\title{
Correction to: Continuous subcutaneous apomorphine infusion in Parkinson's disease: causes of discontinuation and subsequent treatment strategies
}

\author{
Enrica Olivola ${ }^{1}$ - Alfonso Fasano ${ }^{2,3} \cdot$ Sara Varanese $^{4}$ - Francesco Lena ${ }^{1}$ - Marco Santilli ${ }^{1} \cdot$ Cinzia Femiano $^{1}$. \\ Diego Centonze $^{1,5} \cdot$ Nicola Modugno $^{1}$ (D)
}

Published online: 1 July 2019

(C) Fondazione Società Italiana di Neurologia 2019

\section{Correction to: Neurological Sciences 2019}

https://doi.org/10.1007/s10072-019-03920-5

The published version of this article unfortunately contained a mistake in Table 2. CGI-S and CGI-I values has been interchanged. The Table is corrected here.

Publisher's note Springer Nature remains neutral with regard to jurisdictional claims in published maps and institutional affiliations.

The online version of the original article can be found at https://doi.org/ 10.1007/s10072-019-03920-5

Nicola Modugno

nicusmod@gmail.com

1 Unit of Neurology, IRCCS Neuromed, Via Atinense 18, 86077 Pozzilli, IS, Italy

2 The Edmond J. Safra Program in Parkinson's Disease and the Morton and Gloria Shulman Movement Disorders Clinic, Toronto Western Hospital and Division of Neurology, Department of Medicine, University Health Network, Toronto, Ontario, Canada

3 Krembil Research Institute, Toronto, Ontario, Canada

4 Department of Neurology, ASL Lanciano-Vasto-Chieti, S. Pio Hospital, Via C. De Lellis, 66054 Vasto, CH, Italy

5 Department of Systems Medicine, Tor Vergata University, Via Montpellier 1, 00133 Rome, Italy 
Table 2 Clinical features of patients according to the treatment after CSAI

\begin{tabular}{llrrr}
$\begin{array}{l}\text { OTA } \\
(n=58)\end{array}$ & \multicolumn{1}{l}{$\begin{array}{l}\text { DBS } \\
(n=34)\end{array}$} & \multicolumn{1}{l}{$\begin{array}{l}\text { LCIG } \\
(n=16)\end{array}$} & Oral vs. DBS & DBS vs. LCIG \\
\hline $50 \pm 1.6(31-68)$ & $42.16 \pm 1.55(33-58)$ & $49.75 \pm 1.9(31-60)$ & $<0.001$ & 0.029 \\
$61.37 \pm 1.22(41-78)$ & $55.29 \pm 1.34(37-67)$ & $60.75 \pm 2.11(44-74)$ & 0.005 & NS \\
$2.29 \pm 0.29(0.5-9)$ & $2.39 \pm 0.36(0.5-9.2)$ & $3.6 \pm 0.61(0.5-8)$ & $\mathrm{NS}$ & NS \\
$29.35 \pm 0.16(25-30)$ & $29.94 \pm 0.58(28-30)$ & $29.62 \pm 0.27(26-30)$ & 0.033 & NS \\
$27.7 \pm 0.51(10-30)$ & $29.35 \pm 0.21(25-30)$ & $27.18 \pm 0.61(22-30)$ & $\mathrm{NS}$ & NS \\
$2.01 \pm 0.83(1-4)$ & $2.29 \pm 0.89(2-4)$ & $2.43 \pm 0.27(1-6)$ & $\mathrm{NS}$ & NS \\
$5.17 \pm 0.005(5-6)$ & $5.14 \pm 0.61(5-6)$ & $5.31 \pm 0.11(5-6)$ & &
\end{tabular}

Data are expressed as mean $\pm \mathrm{SD}$ (range)

OTA oral therapy alone, DBS deep brain stimulation, $L C I G$ levodopa/carbidopa intestinal gel, PD Parkinson disease, CSAI continuous subcutaneous apomorphine infusion, MMSE Mini-Mental State Examination, CGI-S Clinical Global Impression-Severity, CGI-I Clinical Global ImpressionImprovement 